\title{
Evaluación de las fortalezas del carácter en adolescentes argentinos: validación de una versión breve del VIA-Youth
}

\author{
Nicole Camila Waigel${ }^{1}$ y Viviana Noemí Lemos²
}

\begin{abstract}
RESUMEN
El objetivo del presente trabajo fue, a partir de la versión adaptada por Raimundi et al. (2015) del VIA-Youth (Park \& Peterson, 2006) reelaborar las fortalezas que no presentaban un ajuste psicométrico satisfactorio, seleccionar los mejores items y validar una escala breve para evaluar las fortalezas del carácter en adolescentes. Se llevaron a cabo dos estudios. En el primero se revisaron los items de las dimensiones que lo requerían y las propiedades psicométricas de cada fortaleza (poder discriminativo de los items, estructura interna y consistencia interna). En función de los resultados se seleccionaron los mejores items que conformarian la versión breve preliminar de la escala. En el segundo estudio se evaluaron las propiedades psicométricas de dicha escala, analizando la consistencia interna y recogiendo evidencias de validez de constructo concurrente y factorial (AFE y AFC). La muestra fue no probabilística y estuvo conformada por 963 adolescentes argentinos de entre 12 y 19 años $(M=14.66 ; D E=1.8)$. La misma fue subdividida aleatoriamente en dos submuestras de un $50 \%$ cada una para realizar los análisis factoriales exploratorios y confirmatorios. Todos los ítems resultaron discriminativos y tanto las fortalezas y virtudes como la escala completa mostraron una consistencia interna relativamente adecuada. El AFE indicó la existencia de 6 factores, denominados virtudes según el modelo teórico (coraje, trascendencia, ascendencia, justicia y humanidad, templanza, conocimiento) que explican el 61,8\% de la varianza. El AFC de segundo orden, sin la fortaleza apertura (perteneciente a la dimensión justicia y humanidad), mostró los índices de ajuste más satisfactorios. La escala quedó finalmente compuesta por 66 ítems (22 fortalezas, 6 virtudes). Se concluye que la versión breve definitiva del VIA-Youth presenta adecuadas propiedades psicométricas y posibilita la evaluación de las fortalezas del carácter en adolescentes argentinos de manera práctica, por lo que resulta especialmente útil en contextos de investigación.
\end{abstract}

Palabras clave: virtudes, fortalezas, adolescentes, VIA-YOUTH, escala breve.

\section{Assessment of character strengths in Argentine adolescents: validation of a short version of VIA-Youth}

\begin{abstract}
The objective of the present work was, based on the version adapted by Raimundi et al. (2015) of VIA-Youth (Park \& Peterson, 2006) re-elaborate the strengths that did not
\end{abstract}

\footnotetext{
${ }^{1}$ Centro Interdisciplinario de Investigaciones en Psicología Matemática y Experimental Dr. Horacio J. A. Rimoldi (CIIPME), Consejo Nacional de Investigaciones Científicas y Técnicas (CONICET). Centro Interdisciplinario de Investigaciones en Ciencias de la Salud y del Comportamiento (CIICSAC), Universidad Adventista del Plata. Universidad de Palermo, Argentina; nicolecwaigel@gmail.com; https://orcid.org/0000-0002-3462-081X

2 Centro Interdisciplinario de Investigaciones en Psicología Matemática y Experimental Dr. Horacio J. A. Rimoldi (CIIPME), Consejo Nacional de Investigaciones Científicas y Técnicas (CONICET). Centro Interdisciplinario de Investigaciones en Ciencias de la Salud y del Comportamiento (CIICSAC), Universidad Adventista del Plata, Argentina; viviananoemilemos@gmail.com; https://orcid.org/0000-0002-8855-2293
} 
present a satisfactory psychometric adjustment, select the best items and validate a short scale to assess the strengths of character in adolescents. Two studies were carried out. In the first, the items of the dimensions that required it and the psychometric properties of each strength (discriminative power of the items, internal structure and internal consistency) were reviewed. Based on the results, the best items that would make up the short preliminary version of the scale were selected. In the second study, the psychometric properties of this scale were evaluated, analyzing internal consistency and collecting evidence of validity of the concurrent and factorial construct validity (AFE and AFC). The sample was non-probabilistic and consisted of 963 Argentine adolescents between 12 and 19 years old $(M=14.66 ; S D=1.8)$. It was randomly subdivided into two sub-samples of $50 \%$ each to perform the exploratory and confirmatory factor analyzes. All items were discriminatory, and both strengths and virtues and the full scale showed relatively adequate internal consistency. The EFA indicated the existence of 6 factors, called virtues according to the theoretical model (courage, transcendence, ancestry, justice and humanity, temperance, knowledge) that explain $61.8 \%$ of the variance. The second-order CFA, without the openness strength (belonging to the justice and humanity dimension), showed the most satisfactory adjustment rates. The scale was finally made up of 66 items (22 strengths, 6 virtues). It is concluded that the short definitive version of the VIA-Youth presents adequate psychometric properties and makes it possible to assess the strengths of character in Argentine adolescents in a practical way, being especially useful in research contexts.

Keywords: virtues, strengths, adolescents, VIA-YOUTH, short scale.

Históricamente, el foco de la Psicología estuvo puesto en la evaluación y el tratamiento de los déficits y síntomas psicopatológicos. Sin embargo, a finales del siglo XX surge una nueva propuesta teórica que orienta sus esfuerzos hacia el estudio del desarrollo saludable de las personas a partir de la identificación y comprensión de las características individuales, las experiencias, los entornos y las instituciones que lo posibilitan. Este enfoque, denominado Psicología Positiva, promueve una ampliación del marco de investigación e intervención de la Psicología y considera que los fenómenos positivos de la vida deben ser estudiados y abordados desde una aproximación científica (Park \& Peterson, 2009; Park, Peterson, \& Sun, 2013).

En este contexto, Peterson y Seligman (2004) propusieron el estudio científico de las virtudes y las fortalezas del carácter, también llamadas fortalezas psicológicas. A partir de una exhaustiva revisión de escritos teológicos, filosóficos y culturales, identificaron seis virtudes universales: la sabiduria, el coraje, la justicia, el amor, la templanza y la trascendencia. Debido a que estas resultaban demasiado amplias y abstractas para su estudio, se introdujo el concepto de fortalezas, las cuales son consideradas como la manifestación psicológica de las virtudes.

Las fortalezas se definen como una serie atributos positivos, susceptibles de desarrollo a lo largo de toda la vida, que se manifiestan en los pensamientos, sentimientos y conductas de una persona en diversos ámbitos y a través de distintas situaciones (Peterson \& Seligman, 2004).

Las 24 fortalezas propuestas se agrupan en función de las seis virtudes, siendo esta clasificación una propuesta teórica flexible, susceptible de modificaciones: (a) sabiduria incluye las fortalezas de apertura a la experiencia, creatividad, curiosidad, deseo de aprender y perspectiva; (b) coraje comprende las fortalezas valentía, perseverancia, integridad y vitalidad; (c) amor incluye capacidad de amar, bondad e inteligencia social; (d) justicia agrupa compañerismo, imparcialidad y liderazgo; (e) templanza incluye 
capacidad de perdonar, humildad, prudencia y autorregulación; (f) trascendencia comprende las fortalezas apreciación de la belleza, gratitud, optimismo, humor y espiritualidad (Peterson \& Seligman, 2004).

En la actualidad, se ha profundizado el interés hacia el estudio de las fortalezas $y$ virtudes humanas, con lo cual ha sido necesaria la operacionalización del constructo. A partir de este hecho, se han desarrollado diversos estudios que han demostrado que estas se asocian a un aumento del bienestar psicológico (Giménez, 2010; Grinhauz, 2015) y la satisfacción con la vida (Martínez-Martí \& Ruch, 2017), mejoran el rendimiento académico (Choudhury \& Barooah, 2016), fortalecen la autoestima (Martínez-Martí \& Ruch, 2017) y promueven la resiliencia (Romero, Solis, Beracoechea, \& Andrade, 2019) y los vínculos sociales de calidad (Wagner, 2019), entre otros beneficios.

Debido a la probada asociación entre las fortalezas del carácter y una gran cantidad de factores protectores y promotores de la salud, se ha suscitado un amplio interés por el estudio de este modelo en diversas etapas de la vida y a través de gran cantidad de contextos socioculturales. Vale destacar que, si bien las fortalezas se desarrollan a lo largo de toda la vida, su manifestación en cada etapa del ciclo vital puede variar. Por este motivo, se han diseñado escalas que posibilitan la evaluación de este constructo en diversos grupos etarios (Giménez, Vázquez, \& Hervás, 2010; Park \& Peterson, 2006).

Particularmente, para el estudio de las fortalezas durante la adolescencia se ha diseñado en Estados Unidos el VIA-Youth (Park \& Peterson, 2003, 2006), que evalúa las 24 fortalezas psicológicas propuestas por Peterson y Seligman (2004) a través de 198 ítems. Este instrumento ha sido adaptado en Sudáfrica (Van Eeden, Wissing, Dreyer, Park, \& Peterson, 2008), España (Giménez, 2010), Australia (Toner, Haslam, Robinson, \& Williams, 2012), Alemania y Suiza (Ruch, Weber, Park, \& Peterson, 2014), Brasil (Noronha \& Barbosa, 2016) y Argentina (Raimundi, Molina, Hernández-Mendo, \& Schmidt, 2015).

A través de las diversas adaptaciones se han obtenido soluciones factoriales muy diversas, lo cual da cuenta de la complejidad inherente al constructo que se pretende evaluar (Niemiec, 2013). En linea con lo anterior, Toner et al. (2012) señala que en la mayoría de las adaptaciones disponibles para adultos suelen identificarse entre tres o cuatro factores que engloban las distintas fortalezas, consideradas dentro de este enfoque virtudes; mientras que en las versiones de adolescentes se encuentran entre cuatro o cinco, motivo por el cual se encuentra en discusión el modelo de seis virtudes y el hecho de que exista una única estructura para adultos y adolescentes.

En cuanto a los estudios llevados a cabo en adolescentes de habla hispana, Giménez (2010) llevó adelante la traducción, adaptación y validación del VIA-Youth en población española y encontró asociaciones positivas y significativas entre las fortalezas del carácter y el afecto positivo, la satisfacción con la vida y la autoestima. Por otro lado, observó una relación negativa entre las fortalezas, la depresión y el estrés. Además, halló que las fortalezas cumplen un rol moderador entre el clima familiar percibido y la satisfacción global con la vida.

Otro de los estudios llevados a cabo en España fue el de Ferragut, Blanca y Ortiz-Tallo (2013), quienes revelaron que existe una relación inversa entre las fortalezas del carácter y las actitudes sexistas durante la adolescencia 
temprana, por lo que consideran que la promoción de estos atributos positivos permitiria prevenir la violencia de pareja.

Además, en un estudio longitudinal, Ferragut, Blanca y Ortiz-Tallo (2014) encontraron que las fortalezas se mantienen estables entre los 12 y los 14 años, presentándose un aumento gradual en las puntuaciones de las virtudes Humanidad y Justicia. En cuanto a las diferencias en función del sexo, observaron que las mujeres presentaban puntajes más altos que los varones.

Recientemente, Blanca, Ferragut, Ortiz-Tallo y Bendayan (2018) hallaron asociaciones positivas y significativas entre las fortalezas del carácter y la satisfacción con la vida en adolescentes españoles. Específicamente las fortalezas capacidad de amar y esperanza serian las mejores predictoras de esta relación.

En Argentina, Raimundi et al. (2015) han adaptado el VIA-Youth posibilitando la evaluación de las fortalezas en población adolescente. Más específicamente, Raimundi (2016) trabajó con deportistas adolescentes de alto rendimiento y encontró que las fortalezas que caracterizan a esta población son aquellas que se relacionan con la orientación positiva y la persistencia para alcanzar metas dificiles. Además, según sus hallazgos, las fortalezas optimismo, perseverancia, valentía, vitalidad y religiosidad serian predictoras del flow.

Los estudios mencionados dejan de manifiesto que las fortalezas y virtudes humanas son factores de gran relevancia para el desarrollo óptimo de las personas. Sin embargo, en el estudio científico del desarrollo adolescente ha predominado una conceptualización de esta etapa como un periodo complejo, crítico y traumático de la vida. Son escasos los estudios que evalúan y promueven las cualidades positivas y potencialidades de este grupo etario y durante muchos años se ha minimizado el hecho de que es durante estos años que surgen nuevas formas de pensamiento y de razonamiento moral, se fortalecen las relaciones sociales y se consolida la identidad; todo lo cual constituye una oportunidad para promover las fortalezas del carácter. Reconocer las fortalezas y virtudes durante la adolescencia permitiría reforzar los atributos positivos de los adolescentes, diseñar intervenciones más eficaces y promover sistemas educativos positivos, lo que posibilitaria un desarrollo más pleno (Giménez et al., 2010).

Si bien, como mencionamos anteriormente, existe una versión del VIAYouth adaptada para la Argentina, consideramos importante, por un lado, ajustar las fortalezas que en dicho estudio no obtuvieron propiedades psicométricas satisfactorias $\mathrm{y}$, por otro, proponer una versión breve que permita evaluar dichas fortalezas de una manera más parsimoniosa. Raimundi et al. (2015) remarcan que, aunque su adaptación tiene 83 ítems menos que la escala original, el instrumento resultó demasiado largo al ser aplicado en población adolescente. Además, cuando este tipo de escalas se utilizan con fines de investigación, suelen ser administradas junto con una batería de pruebas, con lo cual contar con una versión breve favorecería su administración, minimizando el sesgo de respuesta relacionado con el cansancio del respondiente (González-Arratia, López-Fuentes, DomínguezEspinosa, \& Torres-Muñoz, 2019).

En vista de los antecedentes expuestos, el objetivo general de este trabajo fue ajustar y validar una escala breve que permita evaluar las fortalezas del carácter en población adolescente, tomando como base la escala adaptada por Raimundi et al. (2015). Se llevaron a cabo dos estudios. El primero tuvo como 
objetivo evaluar la estructura interna de cada una de las fortalezas incluidas en la escala y seleccionar los mejores ítems, proponiendo una versión breve preliminar de la escala. El segundo, analizar las propiedades psicométricas de la mencionada escala y proponer una versión breve definitiva, comparando dos modelos de ajuste.

\section{MÉTODO}

La presente investigación fue de carácter instrumental, con un diseño no experimental y transversal (Montero \& León, 2007; Servera \& Cardo, 2006).

\section{ESTUDIO 1}

\section{Objetivo}

El objetivo del presente trabajo fue, a partir de la versión adaptada por Raimundi et al. (2015) del VIA-Youth (Park \& Peterson, 2006), reelaborar los items de las fortalezas que no presentaban un ajuste psicométrico satisfactorio y seleccionar los mejores ítems de la versión revisada, proponiendo una versión breve preliminar de la escala.

\section{MÉTODO}

\section{Participantes}

Para la revisión de los ítems reelaborados participaron 11 jueces expertos especialistas en el área de psicología positiva, psicologia infanto-juvenil y psicometria y una muestra piloto de 18 adolescentes con edades que oscilaron entre 12 y 18 años $(M=15.33 ; D E=1.5)$.

Seguidamente, se administró la versión revisada a una muestra no probabilística conformada por 963 adolescentes argentinos (373 hombres y 590 mujeres), con edades comprendidas entre 12 y 19 años $(M=14.66 ; D E=$ 1.8). Los participantes concurrian a escuelas secundarias, de gestión pública y privada, de zonas urbanas de las provincias de Entre Ríos y Buenos Aires.

\section{Instrumento}

$\mathrm{El}$ instrumento en revisión fue el Inventario de Fortalezas en Adolescentes (VIA-Youth) desarrollado originalmente por Park y Peterson $(2003,2006)$ y adaptado en Argentina por Raimundi et al. (2015). Si bien la versión original del VIA-Youth (Park \& Peterson, 2003, 2006) evalúa 24 fortalezas del carácter, la versión adaptada por Raimundi et al. (2015) operacionaliza 23 de ellas (dejando de lado inteligencia social) a través de 115 items que se responden por medio de una escala tipo Likert de cinco puntos (desde muy diferente a mí a muy parecido a $m \hat{\imath}$. Este instrumento obtuvo adecuados índices de validez y confiabilidad en la mayoría de sus subescalas (los Alpha oscilan entre .62 y .88) (Raimundi et al., 2015). Las fortalezas que manifestaron dificultades psicométricas fueron: gratitud, por presentar baja confiabilidad e integridad, prudencia, autorregulación, apertura mental y perspectiva, por obtener baja comunalidad. Con el objetivo de ajustar psicométricamente las mencionadas fortalezas, se redactaron nuevos ítems. La versión de la escala a estudiar quedó compuesta por los 115 ítems originales más los 55 incluidos en el presente estudio. 


\section{Procedimientos de recolección de datos}

Los jueces expertos fueron convocados a participar por medio de un correo electrónico, en el cual se les solicitó que evalúen la adecuación sintáctica y semántica de los ítems, la redacción en función del grupo etario destinatario y la coherencia teórica del contenido con la dimensión correspondiente.

Por su parte, los adolescentes de la muestra piloto fueron seleccionados por conveniencia, buscando que estuvieran representadas todas las edades del rango propuesto. Cada uno fue entrevistado individualmente y recibió una copia de la escala con la consigna de leer los items atentamente y expresar si los mismos eran comprensibles. Además, se les solicitó que marquen aquellas palabras cuyo significado no conocían.

Para seleccionar la muestra de tipificación se realizó un muestreo no probabilístico intencional. En primer lugar, se contactó con las escuelas, se solicitó el permiso de los directivos y se explicó el objetivo de la investigación. Seguidamente, se solicitó el consentimiento informado de los padres y/o tutores. La participación de los adolescentes fue voluntaria, se garantizó el anonimato de los mismos y la confidencialidad de los datos obtenidos. La recolección fue realizada de manera grupal en el aula, con una duración aproximada de 30 minutos por curso.

\section{Procedimientos para el estudio psicométrico}

En primer lugar, se revisó la escala adaptada para adolescentes argentinos (Raimundi et al., 2015) prestando especial atención a cada fortaleza y evaluando, en función de lo que mencionan los autores y de los datos psicométricos disponibles, en cuáles se debía ajustar su operacionalización. Se decidió ajustar psicométricamente aquellas que habían presentado baja confiabilidad (gratitud) y baja comunalidad (gratitud, integridad, prudencia, autorregulación, apertura mental y perspectiva). Se redactaron items para cada una de las mencionadas subescalas, tomando como base los propuestos por los autores de la versión original de la escala (Park \& Peterson, 2006), la versión española para adolescentes (Giménez, 2010) y las versiones argentinas para niños y adultos (Cosentino \& Castro Solano, 2015; Grinhauz \& Castro Solano, 2014).

En segunda instancia, para el estudio de los nuevos ítems propuestos, los reactivos fueron sometidos a revisión por parte de jueces expertos. A cada especialista se le envió la definición conceptual de cada una de las fortalezas y los reactivos correspondientes, especificando la fortaleza que pretendian operacionalizar. Luego, la versión revisada a partir de las sugerencias de los jueces fue administrada a una muestra piloto de adolescentes. A continuación, la versión revisada en función de los procedimientos anteriores fue administrada a la muestra de tipificación.

En función de las respuestas brindadas por los 963 adolescentes, se llevaron a cabo diferentes análisis descriptivos de los items. Dado el bajo número de casos perdidos, el criterio de imputación fue reemplazar por la media de la variable. Seguidamente, se evaluó la capacidad discriminativa de cada ítem a través del índice de homogeneidad corregido.

Es necesario destacar que en todas las adaptaciones disponibles del instrumento se consideró que los ítems de cada fortaleza se agrupaban entre sí midiendo lo que se pretende, aunque sin pruebas empíricas de ello. Arias (2008) sostiene que cada parcela (en este caso, cada fortaleza) hace referencia 
a la puntuación total de un conjunto de ítems homogéneos, que se caracterizan por ser unidimensionales. Por este motivo, es necesario demostrar que los ítems de cada fortaleza miden un solo constructo. De este modo, para el estudio de la estructura interna de cada una de las fortalezas se realizaron diferentes Análisis Factoriales Exploratorios (AFE), utilizando el software IBM Statistical Package for the Social Sciencies (SPSS) en su version 21.0. Como requisito previo, se comprobó la factibilidad de realizar estos análisis a través del índice de adecuación muestral Kaiser-Meyer-Olkin (KMO) y la prueba de esfericidad de Barttlet. Para seleccionar los tres items de cada fortaleza que formarian parte de la versión breve del instrumento, se escogieron aquellos que mostraron una buena capacidad de discriminación, aporte a la consistencia interna y mayor saturación en el factor. Finalmente, se calculó la consistencia interna de cada fortaleza a partir de los coeficientes Alpha de Cronbach y Omega de McDonald (McDonald, 1999; Ventura-León \& Caycho Rodríguez, 2017).

\section{RESULTADOS}

\section{Validez de contenido}

Con el objetivo de ajustar psicométricamente las fortalezas, se redactó un pool preliminar de 88 items. Los mismos, como fue mencionado anteriormente, fueron evaluados por 11 jueces expertos. A partir de dicha revisión, se seleccionaron aquellos items que fueron valorados por los jueces como más apropiados con relación al contenido y su adecuación a la población adolescente. De este modo, quedaron seleccionados 55 items, en algunos de los cuales fueron modificadas ciertas expresiones menores. Por ejemplo, la alternativa "Me esfuerzo por terminar todo lo que empiezo y no dejar cosas a medias" fue reemplazada por "No dejo cosas a medias, termino todo lo que empiezo" y "Defiendo lo que está bien, aun cuando tengo miedo" se cambió por "Defiendo lo que creo que está bien, aun cuando pueda traerme inconvenientes".

Seguidamente, los 55 reactivos reelaborados fueron evaluados por una muestra piloto de adolescentes. Dado que los adolescentes no reportaron dificultades en cuanto a la comprensión y claridad de cada uno de los enunciados, estos fueron incluidos en la versión que se administró a la muestra de tipificación. Es decir, la versión administrada quedó conformada por 170 ítems, los 115 de la adaptación de Raimundi et al. (2015) más los nuevos 55.

\section{Análisis descriptivos de los ítems}

Los valores de asimetría y curtosis no superaron en su gran mayoría los guarismos de $+/-2$, a excepción del ítem 58. Por otro lado, se evaluaron los casos perdidos y en ningún caso la respuesta en blanco excedió el $2 \%$, excepto en el ítem 43, el cual no fue contestado por 23 adolescentes (menos de un 3\%). Los mismos fueron imputados a partir del promedio obtenido por los participantes en el ítem.

\section{Poder discriminativo de los ítems}

Se observó que todos los items presentaron correlaciones ítem-total corregidas superiores a .30, lo cual indica que todos presentan una adecuada capacidad de discriminación (Martínez Arias, 2005). 


\section{Validez de constructo}

A continuación, se realizaron los AFE de cada una de las fortalezas que componen el instrumento. En todos los casos el test de Bartlett resultó significativo $(p<.05)$ y el KMO presentó valores adecuados $(>.70)$. A partir de los resultados arrojados, se observó que los ítems se agruparon unidimensionalmente de acuerdo a la fortaleza hipotetizada, presentando en todos los casos un solo factor sin forzar su extracción, a excepción de gratitud, que bajo el criterio de autovalor uno se desdoblaba y hubo que forzar la extracción a un factor.

Los AFE por fortaleza permitieron no solo evaluar la unidimensionalidad de las mismas, sino también depurar la cantidad de items. Fueron seleccionados los tres ítems de cada una de las 23 fortalezas que presentaron mayor saturación en el factor (por encima de .66 en todos los casos). En esta etapa se eliminaron 101 de los 170 ítems, por lo que la versión preliminar de la escala breve quedó conformada por 69 reactivos ${ }^{1}$.

\section{Confiabilidad}

La consistencia interna de cada una de las fortalezas se estudió mediante los coeficientes Alpha de Cronbach y Omega de McDonald. Con relación al Alpha, todas las fortalezas presentaron coeficientes entre .63 y .83. Así mismo, al analizar el coeficiente Omega se encontraron valores superiores a .70 en todos los casos (entre .74 y .89).

\section{ESTUDIO 2}

\section{Objetivo}

El objetivo del segundo estudio fue evaluar el funcionamiento psicométrico de la versión preliminar de la escala breve. Los objetivos específicos fueron: (a) realizar un $\mathrm{AFE}$ de segundo orden, partiendo de las fortalezas, para evaluar cómo las mismas se agrupan en las diferentes virtudes, (b) verificar la estructura hallada realizando un Análisis Factorial Confirmatorio (AFC), (c) evaluar la consistencia interna de la escala y (d) obtener evidencias de validez concurrente evaluando la posible relación entre las fortalezas del carácter y el bienestar psicológico, bajo la hipótesis de que ambos constructos deberian correlacionar de forma positiva y significativa (Giménez, 2010; Harzer, 2016; Toner et al., 2012).

\section{MÉTODO}

\section{Participantes}

Para el estudio de la estructura interna de la escala, la muestra se subdividió de forma aleatoria en dos subgrupos. El primero de ellos fue utilizado para realizar el AFE de segundo orden y estuvo compuesto por 477 adolescentes (181 varones y 296 mujeres), con edades entre 12 y 19 años $(M=$ $14.54 ; D E=1.76)$. Por su parte, con el segundo subgrupo se realizó el AFC para verificar la estructura hallada en el análisis anterior. En este caso, la submuestra estuvo conformada por 486 adolescentes (192 varones y 294 mujeres), con edades entre 12 y 19 años $(M=14.78 ; D E=1.84)$. Los adolescentes, en ambos casos, asistian a escuelas de nivel secundario de las 
provincias de Entre Ríos y Buenos Aires, tanto de gestión pública como privada.

Para el estudio de la validez concurrente, una submuestra de 341 adolescentes (147 varones y 194 mujeres) de la provincia de Buenos Aires, además de haber completado la escala en estudio, respondieron a la Escala de Bienestar Psicológico (Meier \& Oros, 2019). La edad promedio fue de 14.71 años $(D E=1.73)$.

\section{Instrumentos}

Se administró la versión preliminar de la escala breve de fortalezas del carácter derivada del estudio anterior, conformada, como ya se mencionó, por 69 reactivos que operacionalizan 23 fortalezas del carácter y que se responden a través de una escala Likert de cinco puntos (desde muy diferente a mí a muy parecido $a \mathrm{mi}$ ). Adicionalmente, para el estudio de la validez concurrente, se aplicó la versión española de la Escala de Bienestar Psicológico de Ryff, adaptada para población adolescente argentina por Meier y Oros (2019). Este instrumento evalúa el bienestar psicológico a través de 20 ítems que se responden por medio de una escala tipo Likert de cinco puntos (desde Totalmente en desacuerdo hasta Totalmente de acuerdo) y se agrupan en cuatro dimensiones: autoaceptación, crecimiento personal y propósito en la vida, autonomia, y relaciones positivas con otras personas. El funcionamiento psicométrico en cuanto a la validez y confiabilidad resultó satisfactorio en adolescentes argentinos. La consistencia interna de las subescalas osciló entre coeficientes Alpha de .64 y .77. Las dimensiones propuestas que emergieron en el AFE pudieron ser verificadas a través de un AFC con adecuados indices de ajuste (Meier \& Oros, 2019).

\section{Procedimientos para la recolección y el análisis de los datos}

Se utilizaron los mismos procedimientos de recolección de datos seguidos en el Estudio 1.

Para el estudio de la estructura interna de la escala se examinó si la matriz era factorizable a través del índice de adecuación de la muestra de Kaiser-Meyer-Olkin (KMO) y de la prueba de esfericidad de Barttlet. A continuación, se llevó a cabo el AFE a través del programa FACTOR 10.9.02 (Lorenzo-Seva \& Ferrandi, 2015) con el método de ULS con estimación robusta, el cual parte de matrices de correlaciones policóricas, respetando la naturaleza ordinal de las variables. Este fue el método de extracción también seguido en la adaptación del instrumento realizada por Raimundi et al. (2015). Se utilizó una rotación oblicua (Oblimin), partiendo de la hipótesis de que los factores se encuentran correlacionados y siguiendo el tipo de rotación utilizado en el estudio de Raimundi, et al. (2015). Para la extracción de los factores se tuvo en cuenta el criterio de Kaiser (autovalores > 1), el gráfico de sedimentación y el criterio teórico, según el cual se hipotetizaba que las fortalezas se agruparian en seis virtudes.

Seguidamente, para la verificación de la estructura, se realizó un AFC mediante el programa Lisrel en su versión 8.8 (Jöreskog \& Sörborn, 1993). La estimación se efectuó con el método ULS con estimación robusta, en línea con el AFE realizado previamente. El modelo fue evaluado mediante varios índices de bondad de ajuste: Chi-Cuadrado $\left(\chi^{2}\right) ; \chi^{2}$ sobre grados de libertad, para el cual valores inferiores a 3 son deseables (Kline, 2011); RMSEA (<.06), SRMR $(\leq .08)$, CFI ( $\geq .95)$ (Greiff \& Heene, 2017), GFI, NFI y AGFI. Para estos últimos 
indices, valores por encima de .90 indican un ajuste aceptable y valores mayores a .95 un ajuste óptimo (Escobedo Portillo, Hernández Gómez, Estebané Ortega, \& Martínez Moreno, 2016).

Para examinar la confiabilidad de la escala breve definitiva se calculó el coeficiente Omega para cada una de las virtudes.

Por último, para estudiar la validez concurrente se estimaron las correlaciones ( $r$ de Pearson) entre cada una de las virtudes de la versión breve definitiva del VIA-Youth y las dimensiones de la Escala de Bienestar Psicológico de Ryff (Autoaceptación, Relaciones positivas con otras personas, Autonomía, Crecimiento personal y propósito en la vida) (Meier \& Oros, 2019).

\section{RESULTADOS}

\section{Análisis Factorial Exploratorio}

Con respecto a la adecuación de la matriz, el test de Bartlett presentó resultados significativos $\left(\chi^{2}=4094.2 ; g l=253 ; p=.000\right)$ y el KMO fue de .86 , por lo que se consideró pertinente realizar el AFE. El análisis arrojó seis factores sin forzar. El criterio Kaiser y el gráfico de sedimentación indicaron la presencia de seis factores que explicaron el $61.8 \%$ de la variancia. Este número concuerda con el número de factores propuesto por los autores originales de la escala (Park \& Peterson, 2006), aunque la composición de cada uno de ellos fue diferente. En la tabla 1 se resaltan las fortalezas que conformaron cada factor. Los criterios seguidos para esto fueron empíricos y teóricos (Loli, Soto, Lara, \& Fleming, 2016; Merino \& Grimaldo, 2011). Como puede observase, las fortalezas gratitud, perseverancia y capacidad de perdonar presentaron cierta complejidad factorial. Cabe mencionar que es esperable algún grado de complejidad factorial cuando los constructos a operacionalizar sean, como en este caso, muldimensionales (Dominguez-Lara, 2018).

En el caso de gratitud, se observó cierta complejidad factorial entre los factores 1 y 2, pero, debido a que la diferencia entre las saturaciones simultáneas fue mayor a .10, se decidió mantenerlo en el factor de mayor saturación (Meier \& Oros, 2019; Oros, 2014).

En el caso de perseverancia, se pesó tanto en el factor 1 como en el 5, decidiéndose dejarla en el factor 5 dada la concordancia teórica con las otras dos fortalezas que saturaron en dicho factor (prudencia y autorregulación). En cuanto a capacidad de perdonar, la complejidad factorial observada podría explicarse teóricamente, ya que la capacidad de perdonar podría vincularse a los aspectos que engloba el factor 2 (vitalidad, optimismo, capacidad de amar y religiosidad) como a los del factor 4 (apertura mental, equidad y humildad). De todos modos, se decidió dejarla en el factor 4, ya que la saturación en dicho factor fue algo superior.

El primer factor agrupó las fortalezas: solidaridad, valentía, perspectiva, integridad y gratitud y recibió el nombre de coraje. El segundo factor incluyó: vitalidad, optimismo, capacidad de amar y religiosidad y se denominó trascendencia. Por su parte, el tercer factor incluyó las fortalezas: liderazgo, trabajo en equipo y sentido del humor y fue nombrado: ascendencia. El cuarto fue llamado justicia y humanidad y quedó compuesto por: apertura mental, equidad, humildad y capacidad de perdonar. En el quinto factor saturaron las dimensiones de: prudencia, autorregulación y perseverancia y se llamó 
templanza. Por último, el sexto factor quedó conformado por las fortalezas de: curiosidad, creatividad, amor al conocimiento y apreciación de la belleza y recibió la denominación conocimiento.

Tabla 1.

Matriz factorial y peso de cada ítem en el factor.

\begin{tabular}{|c|c|c|c|c|c|c|}
\hline \multirow[b]{2}{*}{ Fortalezas } & \multicolumn{6}{|c|}{ Factores } \\
\hline & 1 & 2 & 3 & 4 & 5 & 6 \\
\hline Solidaridad & .61 & .24 & -.03 & .19 & -.18 & .10 \\
\hline Valentía & .52 & .00 & .07 & -.14 & .06 & .19 \\
\hline Perspectiva & .45 & .01 & .16 & .02 & .01 & .13 \\
\hline Integridad & .45 & -.01 & .00 & .07 & .27 & .02 \\
\hline Gratitud & .43 & .32 & .07 & .06 & .07 & .08 \\
\hline Vitalidad & -.14 & .70 & .33 & .02 & .00 & .12 \\
\hline Optimismo & .00 & .64 & .14 & -.07 & .14 & .09 \\
\hline Capacidad de amar & .25 & .55 & .08 & .13 & .01 & -.04 \\
\hline Religiosidad & .09 & .53 & -.06 & -.15 & .24 & .01 \\
\hline Liderazgo & .07 & .01 & .68 & .00 & .00 & .06 \\
\hline Trabajo en equipo & .21 & .14 & .49 & .17 & .08 & -.03 \\
\hline Sentido del humor & -.03 & .21 & .36 & -.01 & -.22 & .23 \\
\hline Apertura mental & -.26 & .00 & .01 & .59 & .15 & -.02 \\
\hline Equidad & .23 & .07 & .07 & .56 & .10 & .03 \\
\hline Humildad & .19 & -.21 & .04 & .40 & .01 & .03 \\
\hline Capacidad de perdonar & .06 & .30 & -.16 & .32 & -.04 & .07 \\
\hline Prudencia & .08 & .06 & .02 & .05 & .66 & .08 \\
\hline Autorregulación & -.09 & .08 & -.06 & .20 & .60 & .09 \\
\hline Perseverancia & .42 & .21 & .14 & .14 & .38 & .03 \\
\hline Curiosidad & .05 & -.14 & -.03 & -.01 & -.01 & .79 \\
\hline Creatividad & -.15 & .09 & .20 & -.00 & .08 & .66 \\
\hline Amor al conocimiento & .08 & .09 & .12 & -.06 & .21 & .65 \\
\hline Apreciación de la belleza & .16 & .07 & -.20 & .11 & -.01 & .34 \\
\hline Omega* & .62 & .70 & .52 & .53 & .57 & .71 \\
\hline
\end{tabular}

\section{Análisis Factorial Confirmatorio}

Con la segunda submuestra, se pretendió verificar si el modelo obtenido en el AFE (ver tabla 1) ajusta bien a los datos. Los resultados obtenidos por el AFC sugirieron un ajuste aceptable, aunque la fortaleza apertura mental obtuvo un peso no significativo en el factor al que pertenece (.02). En consecuencia, se decidió eliminar esta fortaleza y correr nuevamente el modelo. Como puede observarse en la tabla 2, si bien ambos modelos tienen indices de ajuste relativamente adecuados, el modelo sin apertura mental presentó valores de ajuste más elevados y un poco menos de error.

Tabla 2.

Índices de ajuste del modelo de seis virtudes con y sin apertura.

\begin{tabular}{lcccccccc}
\hline Modelo & $\boldsymbol{X}^{\mathbf{2}}$ & $\boldsymbol{X}^{\mathbf{2}} \mathbf{g l}$ & GFI & AGFI & NFI & CFI & RMSEA [IC] & SRMR \\
\hline Con Apertura & 801.17 & 3.73 & .94 & .93 & .89 & .92 & $.08[.069-.081]$ & .07 \\
Sin Apertura & 683.31 & 3.54 & .95 & .94 & .90 & .93 & $.07[.067-.078]$ & .07 \\
\hline
\end{tabular}




\section{Consistencia interna}

Se puede observar en la tabla 1 que las seis subescalas presentaron valores de Omega que oscilaron entre .52 y .71.

\section{Validez de constructo concurrente}

Tal como era esperable desde el punto de vista teórico, se observaron correlaciones positivas y significativas entre las virtudes y las dimensiones del bienestar psicológico. Específicamente, las dimensiones del bienestar que más se asociaron a las diferentes virtudes son aquellas consideradas como existenciales por Ryff y Keyes (1995): Crecimiento personal y propósito en la vida (ver tabla 3 ).

Tabla 3.

Correlaciones para evidencia de validez concurrente.

\begin{tabular}{lcccc}
\hline & $\begin{array}{c}\text { Crecimiento y } \\
\text { propósito }\end{array}$ & Autoaceptación & $\begin{array}{c}\text { Relaciones } \\
\text { positivas }\end{array}$ & Autonomía \\
\cline { 2 - 5 } Coraje & $.47^{* *}$ & $.29^{* *}$ & $.14^{* *}$ & $.11^{*}$ \\
Trascendencia & $.50^{* *}$ & $.67^{* *}$ & $.41^{* *}$ & $.27^{* *}$ \\
Ascendencia & $.37^{* *}$ & $.35^{* *}$ & $.19^{* *}$ & $.12^{*}$ \\
Justicia y humanidad & $.22^{* *}$ & .07 & .00 & $.10^{*}$ \\
Templanza & $.48^{* *}$ & $.34^{* *}$ & .04 & $.16^{* *}$ \\
Conocimiento & $.32^{* *}$ & $.16^{* *}$ & .00 & .01 \\
\hline${ }^{*} p<.05 ;{ }^{* *} p<.01$ & & & &
\end{tabular}

\section{CONCLUSIONES Y DISCUSIÓN}

El objetivo del presente trabajo fue realizar el ajuste psicométrico y la validación de una escala breve para evaluar las fortalezas del carácter en adolescentes argentinos.

En primera instancia se aportaron evidencias respecto a la validez de contenido y luego se evaluó el funcionamiento de los ítems. Se observó que todos los reactivos aportan a la consistencia interna y logran discriminar según el grado de manifestación de cada una de las fortalezas evaluadas. La pertenencia de los ítems a la fortaleza correspondiente se estableció siguiendo los procedimientos y sugerencias propuestas en las adaptaciones anteriores (Raimundi et al., 2015). A partir de los resultados, se verificó la unidimensionalidad de las subescalas y se seleccionaron los mejores items para componer la escala breve.

Si bien en la versión breve presentada en este estudio emergió una estructura de seis factores, en consistencia con la propuesta teórica de los autores originales de la escala, se observaron ciertas diferencias en la reagrupación de las fortalezas por factor o virtud. La clasificación teórica de las 24 fortalezas en torno a las seis virtudes nunca se ha podido corroborar a nivel empírico y, debido a esto, no existe acuerdo respecto a la estructura factorial del VIA-Youth (Toner et al., 2012). Algunos autores han sugerido que esta se caracteriza por una marcada homogeneidad y, por lo tanto, seria unidimensional (Van Eeden et al., 2008). Sin embargo, diversos estudios han demostrado su multidimensionalidad (Giménez, 2010; Raimundi et al., 2015; Ruch et al., 2014; Toner et al., 2012). La falta de acuerdo en este punto podría explicarse desde una perspectiva evolutiva, ya que los sistemas de valores aún se encuentran en desarrollo durante la adolescencia (Van Eeden et al., 2008). 
Por otro lado, se debe tener en cuenta que la clasificación original fue realizada a nivel teórico y diseñada para población adulta, por lo que cabría la posibilidad de pensar que no existe una única estructura para adultos y adolescentes (Toner et al., 2012).

Al comparar el agrupamiento de las fortalezas que emergió en el presente trabajo con el propuesto por la escala original (Park \& Peterson 2003, 2006) y la adaptación argentina previa (Raimundi et al., 2015) se observan, tal como era esperado, más similitudes con la última. Las dimensiones conocimiento, trascendencia y justicia y humanidad agrupan las mismas fortalezas, aunque en la versión de Raimundi et al. (2015) esta última virtud incluye también las fortalezas solidaridad y trabajo en equipo. Por otro lado, en las tres versiones comparadas, las fortalezas prudencia y autorregulación parecen ser características de la virtud templanza. Siguiendo con la comparación puede observarse que, si bien la dimensión ascendencia emergida en la versión breve es novedosa, se aproxima a la virtud denominada justicia por Park y Peterson (2003, 2006) y no coincide con ninguna de las virtudes encontradas por Raimundi et al. (2015). Por último, la dimensión coraje no emergió como tal en la adaptación argentina previa y en el agrupamiento surgido en la versión breve coincide con la propuesta original de Park y Peterson $(2003,2006)$ solo en las fortalezas valentía e integridad.

Al verificar la estructura a través de un AFC se observó que todas las fortalezas, excepto apertura mental, presentaron saturaciones aceptables (superiores a .30) y las correlaciones entre los factores oscilaron entre .32 y .70. La fortaleza apertura mental presentó una saturación no significativa en el factor justicia y humanidad, motivo por el que se decidió excluirla. Al comparar los índices de ajuste entre ambos modelos (seis virtudes con y sin apertura mental) considerando los valores de $\chi^{2}, \chi^{2} / g 1$, GFI, AGFI, NFI, CFI, RMSEA y SRMR se observa que, si bien ambos modelos presentan un ajuste relativamente adecuado, el modelo que excluye la dimensión apertura mental resultó mejor. Cabe destacar que Raimundi et al. (2015) informaron que esta misma fortaleza presentó coeficientes de estructura menores a .30 en el AFE, aunque dichos autores decidieron mantenerla en su adaptación y sugirieron la realización de estudios confirmatorios.

Adicionalmente, se recogieron evidencias para el estudio de la validez concurrente del instrumento. Se encontró que las fortalezas del carácter se asocian al bienestar psicológico, en línea con otros trabajos que sugieren que las fortalezas se asocian al bienestar, la satisfacción con la vida y el ajuste psicosocial de los adolescentes (Giménez, 2010; Gillham et al., 2011; Harzer, 2016; Noronha \& Martins, 2016; Shoshani \& Slone, 2013; Toner et al., 2012). Más específicamente, se observa que la virtud trascendencia es aquella que más fuertemente correlaciona con todas las dimensiones del bienestar. Estos resultados coinciden con los de diversos estudios llevados a cabo en población adolescente (Gillham et al., 2011; Shoshani \& Swartz, 2018; Shoshani \& Slone, 2013; Toner et al., 2012). Además, tal como reporta Harzer (2016), no se observó en ningún caso un coeficiente de correlación negativo que revelara una relación nociva entre las virtudes y el bienestar psicológico. En relación con lo anterior, diversos estudios han documentado que las fortalezas psicológicas promueven no solo la adopción de conductas positivas asociadas al bienestar, sino que también protegen a las personas de la depresión y el suicidio (Kim et al., 2018), de la manifestación de actitudes sexistas (Ferragut et al., 2013) y de adicciones tecnológicas como por ejemplo Internet y el 
celular (Choi et al., 2015). Las fortalezas de carácter son fundamentales en el desarrollo y mantenimiento del bienestar y la felicidad y se consideran las llaves para el desarrollo óptimo de los jóvenes (Lambert, Passmore, \& Holder, 2015; Park, 2009).

Entre las limitaciones del estudio se encuentra la complejidad factorial observada en las fortalezas gratitud, capacidad de perdonar y perseverancia. En el caso de gratitud, la complejidad observada no es marcada, por lo que se decidió dejarla en el factor que presentó mayor saturación (Meier \& Oros, 2018) y concordancia teórica. En cuanto a la fortaleza capacidad de perdonar, si bien el desdoblamiento observado no sería deseable, es explicable teóricamente (Loli, Soto, Lara, \& Fleming, 2016; Merino \& Grimaldo, 2011), como fue explicado en la sección de resultados. Sin embargo, en el caso de la fortaleza perseverancia, el desdoblamiento encontrado no parecería responder a un motivo teórico, por lo cual sería recomendable estudiar con mayor profundidad esta fortaleza en este período etario y ajustar su correspondiente operacionalización.

Por otro lado, cabe mencionar que, aunque los índices de error (RMSEA y SRMR) y el valor de CFI de la versión breve definitiva se encontraron un poco por fuera de los indicadores de ajuste óptimos (Greiff \& Heene, 2017), otros autores consideran estos valores dentro de los guarismos que indicarian un ajuste razonable (Cupani, 2012; Harrington, 2009; Kline, 2011). Así mismo, el valor obtenido de $\chi^{2} / g l$ fue un poco superior a los limites recomendados por la literatura (Kline, 2011), aunque no supera el límite de 5 (Escobedo Portillo et al., 2016).

Otra limitación del estudio fue la dificultad que se observó al operacionalizar la fortaleza apertura mental. Esta fortaleza finalmente fue eliminada de la versión breve debido a las dificultades psicométricas que presentó. En relación con esto cabe mencionar que, según la hipótesis de la disrupción (Soto \& Tackett, 2015), ciertos procesos de desarrollo y algunos aspectos de la personalidad experimentan caídas momentáneas a lo largo de la adolescencia (Denissen, Van Aken, Penke, \& Wood, 2013). Más específicamente, se ha demostrado que los niveles de apertura, amabilidad y responsabilidad disminuyen al comienzo de la adolescencia para luego aumentar durante la adultez temprana (Soto, John, Gosling, \& Potter, 2011). En esta misma línea, Gander, Hoffman, Proyer y Ruch (2019) y Brown, Blanchard y McGrath (2020) sostienen que probablemente las fortalezas del carácter, al igual que ciertos factores de la personalidad, manifiestan una variación sistemática a lo largo de la adolescencia para luego estabilizarse en la edad adulta. Lo mencionado anteriormente podria explicar las dificultades que se manifestaron en torno a la operacionalización de la fortaleza apertura mental en población adolescente. Se recomienda para futuros estudios profundizar en el constructo apertura mental en esta etapa del ciclo vital, evaluando el modo más adecuado de operacionalizarlo.

Así mismo, otra limitación del estudio es que no se ha incluido en la adaptación argentina previa (Raimundi et al., 2015) ni en la versión breve, la fortaleza inteligencia social. Esta fortaleza fue eliminada de la adaptación llevada a cabo por Raimundi et al. (2015) debido a las dificultades que presentó al momento de ser definida y operacionalizada por los autores originales de la escala (Peterson \& Seligman, 2003, 2006) y evaluada en población adolescente (Giménez, 2010; Raimundi et al., 2015). Silvera, Martinussen y Dahl (2001) consideran que existen dificultades en torno al 
constructo de inteligencia social, ya que se da un solapamiento con otros constructos que ha provocado confusión entre los investigadores con respecto a su definición y desacuerdo respecto a la naturaleza unidimensional o multidimensional del constructo. Las dificultades mencionadas explicarian la complejidad psicométrica de los ítems propuestos para la evaluación de esta fortaleza.

Además, actualmente se sabe que la inteligencia social tiene sus fundamentos en la Teoría de la mente, es decir, en la habilidad de interpretar la conducta de los demás y atribuirles estados mentales, empleando dicha información para predecir y explicar el comportamiento ajeno (Yeh, 2013). El desarrollo y la consolidación de las habilidades de la Teoría de la mente suponen cierto grado de desarrollo de las funciones ejecutivas de alto orden, que maduran de manera diferencial a lo largo de la infancia y la adolescencia (Korzeniowski, 2011). El desarrollo aún en curso durante la adolescencia de las funciones ejecutivas, sumado a las dificultades conceptuales en torno al constructo de inteligencia social, podrían explicar las dificultades en torno a la evaluación de esta fortaleza.

En la actualidad, estamos trabajando en la operacionalización del constructo inteligencia social en adolescentes, con el objetivo de, posteriormente, poder evaluar su comportamiento psicométrico dentro de la versión breve del VIA-Youth.

Por otro lado, se recomienda a futuro someter todos los ítems a un análisis factorial exploratorio de primer orden, que permita estudiar la agrupación de estos y conocer si se reúnen de acuerdo con las fortalezas hipotetizadas. Cabe destacar que a pesar de que se pretendió que la muestra del presente estudio sea heterogénea incluyendo adolescentes que concurren tanto a escuelas públicas como privadas, Argentina es un país que presenta características sociodemográficas y culturales diversas. Con relación a esto, se sugiere ampliar la muestra incluyendo adolescentes que residan en diferentes provincias del país y replicar el AFC con el objetivo de contrastar los resultados obtenidos y poder generalizarlos.

Los resultados de este trabajo dejan de manifiesto que la versión breve definitiva del VIA-Youth presenta adecuadas propiedades psicométricas y que, a pesar de presentar ciertas diferencias con la propuesta teórica original de Park y Peterson $(2003,2006)$ en relación con la agrupación de las fortalezas, exhibe una estructura clara y coherente. Futuros estudios deberian incluir nuevos indicadores de validez y confiabilidad que complementen los hallazgos reportados.

En cuanto a las implicancias del trabajo, este estudio amplía la comprensión de la estructura de las fortalezas del carácter y las virtudes en adolescentes argentinos. Se aportan evidencias respecto al funcionamiento psicométrico de una versión breve que permite evaluar de manera válida y confiable el conjunto de las fortalezas del carácter, minimizando el sesgo de respuesta y posibilitando una administración rápida y práctica.

En sintesis, la versión breve definitiva del VIA-Youth desarrollada en el presente estudio manifiesta una consistencia interna adecuada, especialmente para la escala completa, y evidencias de validez de contenido, de constructo concurrente y factorial. 


\section{REFERENCIAS}

Arias, B. (2008). Desarrollo de un ejemplo de análisis factorial confirmatorio con LISREL, AMOS y SAS. Seminario de actualización en Investigación sobre Discapacidad SAID. España: Universidad de Valladolid.

Blanca, M. J., Ferragut, M., Ortiz-Tallo, M., \& Bendayan, R. (2018). Life satisfaction and character strengths in Spanish early adolescents. Journal of Happiness Studies, 19(5), 1247-1260. https://doi.org/10.1007/s10902-017-9865-y

Brown, M., Blanchard, T., \& McGrath, R. E. (2020). Differences in self-reported character strengths across adolescence. Journal of Adolescence, 79, 1-10. https://doi.org/10.1016/j.adolescence.2019.12.008

Choi, S. W., Kim, D. J., Choi, J. S., Ahn, H., Choi, E. J., Song, W. Y., ... Youn, H. (2015). Comparison of risk and protective factors associated with smartphone addiction and Internet addiction. Journal of behavioral addictions, 4(4), 308-314. https://doi.org/10.1556/2006.4.2015.043

Choudhury, S. \& Barooah, I. (2016). Character strengths and academic achievement in undergraduate college students. Indian Journal of Positive Psychology, 7(1), 76-83.

Cosentino, A. C. \& Castro Solano, A. (2015). IVyF: validez de un instrumento de medida de las fortalezas del carácter de la clasificación de Peterson y Seligman (2004). Psicodebate. Psicología, Cultura y Sociedad, 15(2), 99-122. https://doi.org/10.18682/pd.v15i2.343

Cupani, M. (2012). Análisis de Ecuaciones Estructurales: conceptos, etapas de desarrollo y un ejemplo de aplicación. Revista tesis, 2, 186-199.

Denissen, J. J., van Aken, M. A., Penke, L., \& Wood, D. (2013). Self-regulation underlies temperament and personality: An integrative developmental framework. Child Development Perspectives, 7(4), 255-260. https://doi.org/10.1111/cdep. 12050

Dominguez-Lara, S. (2018) Análisis complementario del MBI-SS en el contexto académico colombiano. Psicología CES, 11 (1), 144-148. https://doi.org/10.21615/cesp.11.1.12

Escobedo Portillo, M. T., Hernández Gómez, J. A., Estebané Ortega, V., \& Martínez Moreno, G. (2016). Modelos de ecuaciones estructurales: Características, fases, construcción, aplicación y resultados. Ciencia \& trabajo, 18(55), 16-22. https://doi.org/10.4067/S0718-24492016000100004

Ferragut, M., Blanca, M. J., \& Ortiz-Tallo, M. (2013). Psychological values as protective factors against sexist attitudes in preadolescents. Psicothema, 25(1), 38-42.

Ferragut, M., Blanca, M. J., \& Ortiz-Tallo, M. (2014). Psychological virtues during adolescence: A longitudinal study of gender differences. European Journal of Developmental Psychology, 11(5), 521531. https://doi.org/10.1080/17405629.2013.876403

Gander, F., Hofmann, J., Proyer, R. T., \& Ruch, W. (2019). Character strengths-stability, change, and relationships with well-being changes. Applied Research in Quality of Life, 1-19. https://doi.org/10.1007/s11482-018-9690-4

Gillham, J., Adams-Deutsch, Z., Werner, J., Reivich, K., Coulter-Heindl, V., Linkins, M., ... Seligman, M. (2011). Character strengths predict subjective well-being during adolescence. The Journal of Positive Psychology, 6(1), 31-44. https://doi.org/10.1080/17439760.2010.536773

Giménez, L. (2010). La medida de las fortalezas psicológicas en adolescentes: Relación con clima familiar, psicopatológica y bienestar psicológico (Tesis doctoral). Universidad Complutense de Madrid, España.

Giménez, M., Vázquez, C., \& Hervás, G. (2010). El análisis de las fortalezas psicológicas en la adolescencia: Más allá de los modelos de vulnerabilidad. Psychology, Society \& Education, 2, 97-116. https://doi.org/10.25115/psye.v2i2.438

González-Arratia-López-Fuentes, N., Domínguez-Espinosa A., \& Torres-Muñoz, M. (2019). Evaluación psicométrica de la Escala de Resiliencia para niños mexicanos (GA-RE14). Evaluar, 19(3), 1-19. https://doi.org/10.30878/ces.v26n3a3

Greiff, S. \& Heene, M. (2017). Why psychological assessment needs to start worrying about model fit. European Journal of Psychological Assessment, 33, 313-317. https://doi.org/10.1027/1015$5759 / \mathrm{a} 000450$

Grinhauz, A. S. (2015). El estudio de las fortalezas del carácter en niños: Relaciones con el bienestar psicológico, la deseabilidad social y la personalidad. Psicodebate, 15(1), 43-68. https://doi.org/10.18682/pd.v15i1.483

Grinhauz, A. S. \& Castro Solano, A. (2014). La evaluación de las virtudes y fortalezas del carácter en niños argentinos: adaptación y validación del Inventario de Virtudes y Fortalezas Para Niños (IVyF Niños). Summa Psicológica, 11(1), 115-126. https://doi.org/10.18774/448x.2014.11.133

Harrington, D. (2009). Confirmatory Factor Analysis. Oxford: Oxford University Press. https://doi.org/10.1093/acprof:oso/9780195339888.001.0001

Harzer, C. (2016). The eudaimonics of human strengths: The relations between character strengths and well-being. En J. Vitters $\varnothing$ (Eds), Handbook of eudaimonic well-being (pp. 307-322). Springer, Cham. https://doi.org/10.1007/978-3-319-42445-3_20

Jöreskog, K. G. \& Sörbom, D. (1993). Lisrel 8: Structural equation modeling with the simplis command language. Hilldale, N.J: Lawrence Erlbaum Associates Publishers.

Kim, H. R., Kim, S. M., Hong, J. S., Han, D. H., Yoo, S. K., Min, K. J., \& Lee, Y. S. (2018). Character strengths as protective factors against depression and suicidality among male and female employees. BMC public health, 18(1), 1084. https://doi.org/10.1186/s12889-018-5997-1

Kline, R. B. (2011). Principles and practice of structural equation modeling. Nueva York: Guilford press.

Korzeniowski, C. G. (2011). Desarrollo evolutivo del funcionamiento ejecutivo y su relación con el aprendizaje escolar. Revista de Psicología, 7(13), 7-26.

Lambert, L., Passmore, H. A., \& Holder, M. D. (2015). Foundational frameworks of positive Psychology: Mapping well-being orientations. Canadian $\quad$ Psychology, 56(3), 
https://doi.org/10.1037/cap0000033

Loli, J. S. N., Soto, C. M., Lara, S. A. D., \& Fleming, J. S. (2016). Carta al editor: "Importancia de la evaluación de la simplicidad factorial: Re-análisis a Zicado, Palma y Garrido (2012)". Revista Latinoamericana de Ciencias Sociales, Niñez y Juventud, 14(2), 1682-1684.

Lorenzo-Seva, U., \& Ferrandi, P. J. (2015). FACTOR 10.3 for Windows (64 bits). Tarragona, Spain: Rovira $\mathrm{i}$ Virgili University.

Martínez Arias, R. (2005). Psicometría: Teoría de los test psicológicos y educativos. Madrid: Sintesis.

Martínez-Martí, M. L. \& Ruch, W. (2017). Character strengths predict resilience over and above positive affect, self-efficacy, optimism, social support, self-esteem, and life satisfaction. The Journal of Positive Psychology, 12(2), 110-119. https://doi.org/10.1080/17439760.2016.1163403

McDonald, R. P. (1999). Test theory: A unified approach. Mahwah, NJ: Lawrence Erlbaum.

Meier, L. \& Oros, L. (2019). Adaptación y Análisis Psicométrico de las Escalas de Bienestar Psicológico de Ryff en Adolescentes Argentinos. Psykhe (Santiago), 28(1), 1-16. https://doi.org/10.7764/psykhe.27.2.1169

Merino, C., \& Grimaldo, M. (2011). Complejidad factorial y conductas moralmente controversiales. Revista Argentina de Ciencias del Comportamiento, 3(3), 38-43.

Montero, I., \& León, O. G. (2007). A guide for naming research studies in psychology. International Journal of Clinical and Health Psychology, 7(3), 847-862.

Niemiec, R. (2013). VIA Character strenghts: Research and practice (the first 10 years). En H. Knoop \& A. Delle Fave (Eds.), Well-being and cultures (pp. 11-30). New York: Springer. https://doi.org/10.1007/978-94-007-4611-4_2

Noronha, A. \& Barbosa, A. J. G. (2016). Escala de Forças e Virtudes. Avaliação em Psicologia Positiva, 4253.

Noronha, A. P. P. \& Martins, D. F. (2016). Associations between character strengths and life satisfaction: A study with college students. Acta Colombiana de Psicología, 19(2), 97-103.

Oros, L. B. (2014). Nuevo cuestionario de emociones positivas para niños. Anales de Psicologia/Annals of Psychology, 30(2), 522-529. https://doi.org/10.6018/analesps.30.2.158361

Park, N. (2009). Building strengths of character: Keys to positive youth development. Reclaiming Children and Youth, 18(2), 42.

Park, N. \& Peterson, C. (2003). Assessment of character strengths among youth: The values in Action Inventory of Strengths for Youth. Indicators of Positive Development. Recuperado de http://www.childtrends.org/ Files/Child_Trends-2003_03_12_PD_PDConfPetersonPark.pdf

Park, N. \& Peterson, C. (2006). Moral competence and character strengths among adolescents: The development and validation of the Values in Action Inventory of Strengths for Youth. Journal of adolescence, 29(6), 891-909. https://doi.org/10.1016/j.adolescence.2006.04.011

Park, N. \& Peterson, C. (2009). Achieving and sustaining a good life. Perspective son Psychological Science, 4(4), 422-428. https://doi.org/10.1111/j.1745-6924.2009.01149.x

Park, N., Peterson, C., \& Sun, J. K. (2013). La psicología positiva: investigación y aplicaciones. Terapia psicológica, 31(1), 11-19. https://doi.org/10.4067/S0718-48082013000100002

Peterson, C. \& Seligman, M. (2004). Character strengths and virtues: A handbook and classification. New York: Oxford University Press.

Raimundi, M. J. (2016). Experiencias óptimas en el deporte adolescente de alto rendimiento: Influencia de las fortalezas y el contexto familiar (Tesis de doctorado). Universidad de Málaga-Universidad Nacional de La Plata.

Raimundi, M. J., Molina, M. F., Hernández-Mendo, A., \& Schmidt, V. (2015). Adaptación argentina del Inventario de Fortalezas en adolescentes (VIA-Youth): Propiedades psicométricas y alternativas para su factorización. Revista Iberoamericana de Diagnóstico y Evaluación-e Avaliação Psicológica, 3(45), 159174. https://doi.org/10.21865/RIDEP45.3.13

Romero, N., Solis, M., Beracoechea, L. A., \& Andrade, H. (2019). Fortalezas de carácter como predictoras de resiliencia en adultos mexicanos. Psicodebate. Psicología, Cultura y Sociedad, 1(1), 7-17. https://doi.org/10.18682/pd.v1i1.803

Ruch, W., Weber, M., Park, N., \& Peterson, C. (2014). Character Strengths in Children and Adolescents. European Journal of Psychological Assessment, 30(1), 57-64. https://doi.org/10.1027/1015$5759 / \mathrm{a} 000169$

Ryff, C. D. \& Keyes, C. L. M. (1995). The structure of psychological well-being revisited. Journal of Personality and Social Psychology, 69(4), 719-727. https://doi.org/10.1037/0022-3514.69.4.719

Servera, M. \& Cardo, E. (2006). Children Sustained Attention Task (CSAT): Normative, reliability, and validity data. International Journal of Clinical and Health Psychology, 6(3), 697-707.

Shoshani, A. \& Shwartz, L. (2018). From character strengths to children's well-being: development and validation of the character strengths inventory for elementary school children. Frontiers in psychology, 9, 2123. https://doi.org/10.3389/fpsyg.2018.02123

Shoshani, A. \& Slone, M. (2013). Middle school transition from the strengths perspective: Young adolescents' character strengths, subjective well-being, and school adjustment. Journal of Happiness Studies, 14(4), 1163-1181. https://doi.org/10.1007/s10902-012-9374-y

Silvera, D., Martinussen, M., \& Dahl, T. I. (2001). The Tromsø Social Intelligence Scale, a self-report measure of social intelligence. Scandinavian journal of psychology, 42(4), 313-319. https://doi.org/10.1111/1467-9450.00242

Soto, C. J. \& Tackett, J. L. (2015). Personality traits in childhood and adolescence: Structure, development, and outcomes. Current Directions in Psychological Science, 24, 358-362. https://doi.org/10.1177/0963721415589345

Soto, C. J., John, O. P., Gosling, S. D., \& Potter, J. (2011). Age differences in personality traits from 10 to 
65: Big Five domains and facets in a large cross-sectional sample. Journal of personality and social psychology, 100(2), 330. https://doi.org/10.1037/a0021717

Toner, E., Haslam, N., Robinson, J., \& Williams, P. (2012). Character strengths and wellbeing in adolescence: Structure and correlates of the Values in Action Inventory of Strengths for Children. Personality and Individual Differences, 52(5), 637-642. https://doi.org/10.1016/j.paid.2011.12.014

Van Eeden, C., Wissing, M., Dreyer, J., Park, N., \& Peterson, C. (2008). Validation of the Values in Action Inventory of Strengths for Youth (VIA-Youth) among South African learners. Journal of Psychology in Africa, 18, 143-154. https://doi.org/10.1080/14330237.2008.10820181

Ventura León, J. L. \& Caycho Rodríguez, T. (2017). El coeficiente Omega: un método alternativo para la estimación de la confiabilidad. Revista Latinoamericana de Ciencias Sociales, Niñez y Juventud, 15(1), 625-627.

Wagner, L. (2019). Good character is what we look for in a friend: character strengths are positively related to peer acceptance and friendship quality in early adolescents. The Journal of Early Adolescence, 39(6), 864-903. https://doi.org/10.1177/0272431618791286

Yeh, Z. T. (2013). Role of theory of mind and executive function in explaining social intelligence: A structural equation modeling approach. Aging \& Mental Health, 17(5), 527-534. https://doi.org/10.1080/13607863.2012.758235

${ }^{1}$ Por cuestiones de espacio no se adjunta la lista completa de los ítems de los cuales se partió, pero se encuentra a disposición del lector si lo requiriera. 


\section{ANEXO \\ ESCALA BREVE DE FORTALEZAS PARA ADOLESCENTES (VIA-YOUTH)}

A continuación, hay una lista de afirmaciones que podrían describirte. Por favor, leé cada una y decidí en qué grado refleja cómo sos, marcando con una $\mathbf{X}$ la opción que mejor te represente. No hay respuestas correctas ni incorrectas. Sé tan sincero/a como puedas.

\begin{tabular}{|c|c|c|c|c|c|c|}
\hline \multirow{2}{*}{\multicolumn{2}{|c|}{ 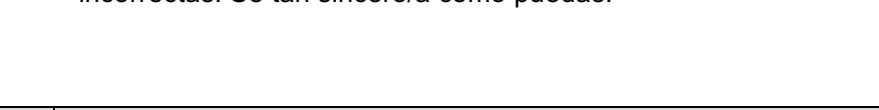 }} & \multirow{3}{*}{$\begin{array}{c}\text { Muy } \\
\text { diferente a } \\
\text { mí }\end{array}$} & \multirow{3}{*}{$\begin{array}{c}\text { Algo } \\
\text { diferente } \\
\text { a mí } \\
\end{array}$} & \multirow{3}{*}{$\begin{array}{c}\text { Ni una } \\
\text { opción ni la } \\
\text { otra }\end{array}$} & \multirow{3}{*}{$\begin{array}{c}\text { Algo } \\
\text { parecido } \\
\text { a mí }\end{array}$} & \multirow{3}{*}{$\begin{array}{c}\text { Muy } \\
\text { parecido } \\
\text { a mí }\end{array}$} \\
\hline & & & & & & \\
\hline 1 & Tengo muchas ideas creativas. & & & & & \\
\hline 2 & $\begin{array}{l}\text { Cuando alguien me ayuda o es bueno conmigo, le hago saber } \\
\text { que estoy agradecido/a. }\end{array}$ & & & & & \\
\hline 3 & Defiendo lo que creo que es correcto, aunque tenga miedo. & & & & & \\
\hline 4 & Una vez que hago un plan, lo cumplo. & & & & & \\
\hline 5 & Suelo sentirme lleno/a de energía. & & & & & \\
\hline 6 & $\begin{array}{l}\text { Cuando sé de personas que necesitan ayuda, me preocupo por } \\
\text { ellos. }\end{array}$ & & & & & \\
\hline 7 & Le doy la misma importancia a las opiniones de todos. & & & & & \\
\hline 8 & $\begin{array}{l}\text { Puedo seguir siendo amigo/a de quienes me trataron mal, si me } \\
\text { piden perdón. }\end{array}$ & & & & & \\
\hline 9 & Soy precavido/a en cualquier cosa que hago. & & & & & \\
\hline 10 & Me encanta el arte, la música, la danza o el teatro. & & & & & \\
\hline 11 & $\begin{array}{l}\text { Siempre tengo esperanza, sin importar lo mal que parezcan las } \\
\text { cosas. }\end{array}$ & & & & & \\
\hline 12 & Los demás dicen que soy muy gracioso/a. & & & & & \\
\hline 13 & $\begin{array}{l}\text { Antes de que mis amigos tomen una decisión importante, suelen } \\
\text { pedir mi opinión. }\end{array}$ & & & & & \\
\hline 14 & Aunque tenga muchas ganas de hacer algo ya, puedo esperar. & & & & & \\
\hline 15 & $\begin{array}{l}\text { Siento interés o curiosidad por las personas, lugares o cosas que } \\
\text { no me son familiares. }\end{array}$ & & & & & \\
\hline 16 & $\begin{array}{l}\text { Cuando hay una oportunidad para aprender cosas nuevas, } \\
\text { participo activamente. }\end{array}$ & & & & & \\
\hline 17 & Miento para conseguir salir de problemas. & & & & & \\
\hline 18 & Suelo decirle a mis amigos y a mis familiares que los quiero. & & & & & \\
\hline 19 & Trabajo muy bien en equipo. & & & & & \\
\hline 20 & Aunque sea realmente bueno/a en algo, no "me la creo". & & & & & \\
\hline 21 & Creo que alguien en el cielo me guiará para hacer lo correcto. & & & & & \\
\hline 22 & Hago todo lo que puedo por ayudar a quien lo necesita. & & & & & \\
\hline 23 & Me gusta crear cosas nuevas o diferentes. & & & & & \\
\hline 24 & $\begin{array}{l}\text { Cuando veo que alguien es malo con los demás, le digo que eso } \\
\text { no está bien. }\end{array}$ & & & & & \\
\hline 25 & Cuando empiezo un proyecto, siempre lo termino. & & & & & \\
\hline 26 & Aunque alguien no me caiga bien, lo trato con justicia. & & & & & \\
\hline 27 & $\begin{array}{l}\text { Cuando hay algún proyecto en grupo para hacer, los demás me } \\
\text { quieren a cargo de él. }\end{array}$ & & & & & \\
\hline 28 & Soy cuidadoso/a a la hora de tomar decisiones. & & & & & \\
\hline 29 & $\begin{array}{l}\text { Cuando veo arte o escucho música, suelo olvidarme cuánto } \\
\text { tiempo pasó. }\end{array}$ & & & & & \\
\hline 30 & Me gusta contar chistes o historias divertidas. & & & & & \\
\hline 31 & Las personas suelen decir que doy buenos consejos. & & & & & \\
\hline 32 & No actúo como si fuera mejor que los demás. & & & & & \\
\hline 33 & $\begin{array}{l}\text { Estoy dispuesto/a a trabajar lo que sea necesario por nuestro } \\
\text { equipo. }\end{array}$ & & & & & \\
\hline 34 & Comparto mis sentimientos con mis amigos o familia. & & & & & \\
\hline 35 & Miento para conseguir lo que quiero. & & & & & \\
\hline 36 & Me encanta aprender cosas nuevas. & & & & & \\
\hline 37 & Cuando pienso en todo lo que tengo, me siento agradecido/a. & & & & & \\
\hline 38 & Hago preguntas sobre las cosas que pasan en el mundo. & & & & & \\
\hline 39 & Cuando rezo/oro, me siento mejor. & & & & & \\
\hline 40 & Cuando juego con otros, quieren que yo sea el/la líder. & & & & & \\
\hline 41 & Suelo sentirme alegre. & & & & & \\
\hline 42 & $\begin{array}{l}\text { Cuando la gente me pide perdón, les doy una segunda } \\
\text { oportunidad. }\end{array}$ & & & & & \\
\hline 43 & Puedo esperar mi turno sin perder la paciencia. & & & & & \\
\hline 44 & $\begin{array}{l}\text { Creo que las cosas siempre saldrán bien, sin importar lo difícil } \\
\text { que parezcan ahora. }\end{array}$ & & & & & \\
\hline
\end{tabular}




\begin{tabular}{|c|c|c|c|c|c|c|}
\hline & & $\begin{array}{c}\text { Muy } \\
\text { diferente a } \\
\text { mí }\end{array}$ & $\begin{array}{c}\text { Algo } \\
\text { diferente } \\
\text { a mí }\end{array}$ & $\begin{array}{c}\text { Ni una } \\
\text { opción ni la } \\
\text { otra }\end{array}$ & $\begin{array}{c}\text { Algo } \\
\text { parecido } \\
\text { a mí }\end{array}$ & $\begin{array}{c}\text { Muy } \\
\text { parecido } \\
\text { a mí }\end{array}$ \\
\hline 45 & Mirar obras de arte o escuchar música linda me hace sentir mejor. & & & & & \\
\hline 46 & Siempre que hago algo, pongo todo mi esfuerzo. & & & & & \\
\hline 47 & Tengo preguntas sobre muchas cosas. & & & & & \\
\hline 48 & Me enfrento a los chicos que actúan mal o injustamente. & & & & & \\
\hline 49 & $\begin{array}{l}\text { Cuando tengo un problema, tengo a alguien que estará ahí por } \\
\text { mí. }\end{array}$ & & & & & \\
\hline 50 & Soy muy cooperativo/a cuando trabajo en grupo. & & & & & \\
\hline 51 & $\begin{array}{l}\text { Tengo cuidado de no hacer cosas de las que me pueda arrepentir } \\
\text { más adelante. }\end{array}$ & & & & & \\
\hline 52 & Soy bueno/a haciendo que una situación aburrida sea divertida. & & & & & \\
\hline 53 & Mis amigos me piden consejos. & & & & & \\
\hline 54 & Aunque alguien no sea bueno conmigo, yo lo trato de forma justa. & & & & & \\
\hline 55 & Creo que la vida es emocionante. & & & & & \\
\hline 56 & Me encanta aprender cómo hacer diferentes cosas. & & & & & \\
\hline 57 & $\begin{array}{l}\text { Cuando me pasan cosas buenas, me siento agradecido/a con la } \\
\text { gente que me ayudó. }\end{array}$ & & & & & \\
\hline 58 & Digo la verdad, aunque me traiga problemas. & & & & & \\
\hline 59 & Soy un/a líder en el/la que los demás confían. & & & & & \\
\hline 60 & No "me la creo" por mis logros. & & & & & \\
\hline 61 & Puedo controlarme aunque esté realmente enojado/a. & & & & & \\
\hline 62 & Aunque alguien me lastime, lo perdono si se disculpa. & & & & & \\
\hline 63 & Soy muy positivo/a con respecto al futuro. & & & & & \\
\hline 64 & Me preocupo por los demás cuando tienen problemas. & & & & & \\
\hline 65 & Tengo fe. & & & & & \\
\hline 66 & Creo que soy muy creativo/a. & & & & & \\
\hline
\end{tabular}

CLAVE DE CORRECCIÓN DE LA ESCALA BREVE DE FORTALEZAS DEL CARÁCTER

\begin{tabular}{|c|c|c|}
\hline $\begin{array}{l}\text { VIRTUD CORAJE (Omega .620) } \\
\text { Solidaridad: } 6,22,64 \\
\text { Valentía: } 3,24,48 \\
\text { Perspectiva: } 13,31,53 \\
\text { Integridad: } 17,35,58 \\
\text { Gratitud: } 2,37,57\end{array}$ & $\begin{array}{l}\text { VIRTUD TRASCENDENCIA } \\
\text { (Omega .703) } \\
\text { Vitalidad: } 5,41,55 \\
\text { Optimismo: } 11,44,63 \\
\text { Capacidad de amar: 18, 34, } 49 \\
\text { Religiosidad: } 21,39,65\end{array}$ & $\begin{array}{l}\text { VIRTUD ASCENDENCIA (Omega } \\
. \mathbf{5 2 7} \text { ) } \\
\text { Liderazgo: } 27,40,59 \\
\text { Trabajo en equipo: } 19,33,50 \\
\text { Sentido del humor: } 12,30,52\end{array}$ \\
\hline $\begin{array}{l}\text { VIRTUD JUSTICIA Y } \\
\text { HUMANIDAD (Omega .539) } \\
\text { Equidad: } 7,26,54 \\
\text { Humildad: } 20,32,60 \\
\text { Capacidad de perdonar: } 8,42,62\end{array}$ & $\begin{array}{l}\text { VIRTUD TEMPLANZA (Omega } \\
\text {.573) } \\
\text { Prudencia: 9, 28, } 51 \\
\text { Autorregulación: } 14,43,61 \\
\text { Perseverancia: } 4,25,46\end{array}$ & $\begin{array}{l}\text { VIRTUD CONOCIMIENTO (Omega } \\
.718 \text { ) } \\
\text { Curiosidad: } 15,38,47 \\
\text { Creatividad: } 1,23,66 \\
\text { Amor al conocimiento: } 16,36,56 \\
\text { Apreciación por la belleza: } 10,29,45\end{array}$ \\
\hline
\end{tabular}

Nota: La puntuación va desde Muy diferente a mí (1) a Muy parecido a mí (5). Los valores de los ítems en negrita deben ser invertidos

Recibido 21-04-2020 | Aceptado 12-08-2020

Este trabajo se encuentra bajo una Licencia Creative Commons Atribución 4.0 Internacional que permite a terceros utilizar lo publicado siempre que se dé el crédito pertinente a los autores y a Psicodebate 